\title{
Working memory capacity of biological movements predicts empathy traits
}

\author{
Zaifeng Gao ${ }^{1} \cdot$ Tian $_{\mathrm{Ye}^{1}} \cdot$ Mowei Shen ${ }^{1} \cdot$ Anat Perry $^{2}$
}

Published online: 15 July 2015

(C) Psychonomic Society, Inc. 2015

\begin{abstract}
Working memory (WM) and empathy are core issues in cognitive and social science, respectively. However, no study so far has explored the relationship between these two constructs. Considering that empathy takes place based on the others' observed experiences, which requires extracting the observed dynamic scene into WM and forming a coherent representation, we hypothesized that a sub-type of WM capacity, i.e., WM for biological movements (BM), should predict one's empathy level. Therefore, WM capacity was measured for three distinct types of stimuli in a change detection task: BM of human beings (BM; Experiment 1), movements of rectangles (Experiment 2), and static colors (Experiment 3). The first two stimuli were dynamic and shared one WM buffer which differed from the WM buffer for colors; yet only the $\mathrm{BM}$ conveyed social information. We found that BM-WM capacity was positively correlated with both cognitive and emotional empathy, with no such correlations for WM capacity of movements of rectangles or of colors. Thus, the current study is the first to provide evidence linking a specific buffer of WM and empathy, and highlights the necessity for considering different WM capacities in future social and clinical research.
\end{abstract}

Electronic supplementary material The online version of this article (doi:10.3758/s13423-015-0896-2) contains supplementary material, which is available to authorized users.

Mowei Shen

mwshen@zju.edu.cn

1 Department of Psychology, Zhejiang University, Zhejiang Hangzhou 310007, China

2 Department of Psychology and Helen Wills Neuroscience Institute, University of California Berkeley, Berkley, CA, USA
Keywords Visual working memory $\cdot$ Social cognition · Working memory

\section{Introduction}

Working memory (WM) is the set of mental processes involved in retaining a small amount of information in a temporarily accessible state for use in ongoing cognitive tasks (Baddeley, 2012). If sufficient information is not retained and integrated into WM, various high-level activities (e.g., reading, language comprehension) cannot be completed (e.g., Baddeley, 2003; Cowan et al., 2005). Although the number of items that can be maintained in WM is limited to roughly four, there are large individual differences in this capacity (for reviews see Luck \& Vogel, 2013; Mance \& Vogel, 2013). Not surprisingly, a correlation has been found between WM capacity and different aptitudes of cognitive human behavior (e.g., attentional capture, intelligence, etc.; Cowan et al., 2005; Fukuda \& Vogel, 2009; Unsworth, Fukuda, Awh, \& Vogel, 2014). These studies serve as an important step in our understanding of the basic determinants of the examined aptitudes.

WM studies have documented that although human biological movements (BM) stimuli share a memory buffer with other dynamic stimuli, they are stored independently from colors, shapes, and locations (e.g., Shen, Gao, Ding, Zhou, \& Huang, 2014; Smyth \& Pendleton, 1990; Wood, 2007). The ability to perceptually process BM is suggested as a hallmark of social cognition (Pavlova, 2012), and the BM-WM buffer is suggested to play an important role in transferring ongoing social information from perception to WM (e.g., Shen et al., 2014; Urgolites \& Wood, 2013). Recently, Gao et al. (2014) revealed that the human mirror neuron system (hMNS) (e.g., Baird, Scheffer, \& Wilson, 2011; Ulloa \& Pineda, 2007), indexed by EEG mu suppression (8-12 Hz), 
plays a pivotal role in rehearsing BM information (but not non-biological information) in WM, strengthening the notion that rehearsing BM in WM relies, at least in part, on an independent mechanism. Just as WM for general visual objects (e.g., colors) has been linked to general cognitive abilities such as intelligence, we hypothesized that WM capacities that are specific to BM would correlate with social functioning.

We focused on the correlation between WM of BM and empathy, an essential aspect of normal social functioning, which has been extensively studied in the last decades from developmental, social, clinical, and neuroscience perspectives (e.g., Baron-Cohen \& Wheelwright, 2004; Batson et al., 1997; Blakemore, 2008; Decety \& Jackson, 2004; Knafo et al., 2008; Shamay-Tsoory, 2011). Empathy, broadly defined, refers to the cognitive as well as the emotional reactions of an individual to the observed experiences of other individuals (Shamay-Tsoory, 2011). It not only allows us to understand what someone else might be thinking and, thus, predict their behavior (i.e., cognitive empathy), but also enables us to tune into their feelings, or experience affective reactions to the observed experiences of others (i.e., emotional empathy) (Shamay-Tsoory, 2011; Zaki, 2014). Previous studies have revealed that cognitive empathy is related to cognitive flexibility (e.g., Eslinger, 1998; Shamay-Tsoory, Tomer, Goldsher, Berger, \& Aharon-Peretz, 2004), a key function of central executive of WM (c.f., Baddeley, 2012), implying that WM plays a role in empathy (see also Decety \& Jackson, 2004). However, to the best of our knowledge, no study has directly explored the relationship between empathy and WM.

Here we argue that a prerequisite for inducing empathy is to understand what is taking place, requiring the individual to extract the observed social dynamic scene into WM and form a coherent representation from it (Henderson \& Hollingworth, 2003). Considering that performance on human BM perception tasks is considered as a critical index reflecting social cognition (see Pavlova, 2012, for a review), we further reasoned that the more BM information one can store, the easier it is for him/her to extract social cues from the environment and from that to understand others' emotions and intentions. Thus, we hypothesized that empathetic abilities would correlate with WM for BM. Revealing the link between empathic abilities and WM for BM has important implications for our understanding of both constructs, and may have clinical implications for people with social cognitive deficits.

To this end, we measured WM capacity related to three distinct types of information: BM of human beings (Experiment 1), movements of rectangles (Experiment 2), and static colors (Experiment 3). Following each experiment, we measured the empathy level of the participants using two empathy questionnaires. We predicted that only WM capacity for BM would correlate with empathy, but other types of information (e.g., movements of rectangle, colors) would not.

\section{Methods}

\section{Participants}

Experiments 1, 2, and 3 had 66 (39 males; mean \pm SD age $20.23 \pm 2.78$ years $),{ }^{1} 40$ (17 males; mean \pm SD age $21.22 \pm$ 1.57 years ), and 40 (18 males; mean \pm SD age $21.57 \pm$ 2.41 years) participants, respectively (Simmons, Nelson, \& Simonsohn, 2011). Nine, four, and three participants in Experiments 1, 2, and 3, respectively, were excluded from further analyses because their score on at least one questionnaire was beyond two SDs of the tested group. All participants were students at Zhejiang University, who provided signed informed consent and had normal color vision and normal or corrected-to-normal visual acuity. They received payment/ course credit for their participation. Each participant only took part in one experiment. The study was approved by the Research Ethics Board of Zhejiang University.

\section{Stimuli and apparatus}

The stimuli were created and presented on a grey $(128,128$, 128 in RGB value) background on a 17-in CRT monitor.

Point-light displays (PLDs) were used as the BM stimuli (Johansson, 1973). The PLDs depict human activity through a simple set of light points (e.g., 12 points), which are placed at distinct joints of a moving human body. Although highly impoverished (e.g., texture and form cues are absent), once in motion, these PLDs are rapidly recognized as coherent, meaningful movements. The PLDs can minimize the availability of structural cues and isolate BM information from other sources (e.g., color, contour, and texture; for a review see Troje, 2013). The PLDs were selected from the Vanrie and Verfaillie (2004) database: cycling, jumping, painting, spading, walking, waving, and chopping (see Fig. 1A). The movements of the rectangles in Experiment 2 were created based on a 12-dotted rectangle. The shape of a rectangle was chosen because the PLDs used in Experiment 1 exhibited a global appearance of a rectangle. Moreover, in line with the BM stimuli in Experiment 1, the dotted rectangle in Experiment 2 had seven distinct movements (see Fig. 1B and Supplementary video). Every animation in Experiments 1 and 2 consisted of 30 distinct frames, and each frame was displayed twice in succession, leading to a 1-s animation (refresh rate, $60 \mathrm{~Hz}$ ). The displays subtended a visual angle of approximately $1.64^{\circ} \times 1.64^{\circ}$ from a viewing distance of $60 \mathrm{~cm}$. Following previous studies measuring BM capacity (e.g., Shen et al., 2014), one to five distinct stimuli were randomly

\footnotetext{
${ }^{1}$ To examine the robustness of the key result, we first collected data from 40 participants, and after 6 months we collected data from 26 new participants. We analyzed these two sets separately in the Appendix, and in both obtained similar results.
} 


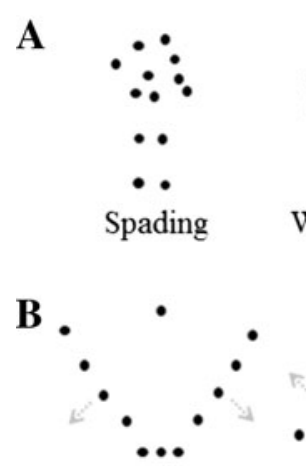

(1)
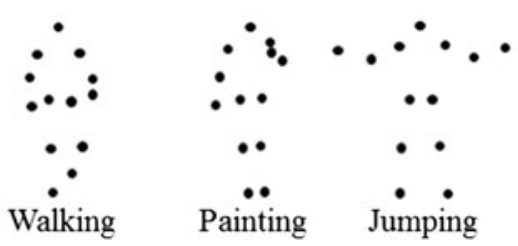
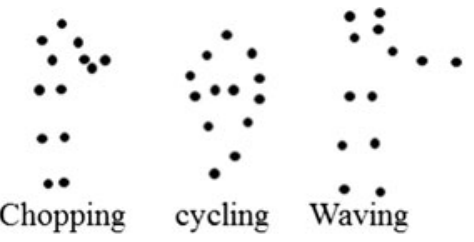

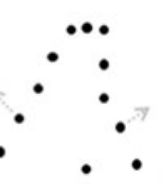

(2)

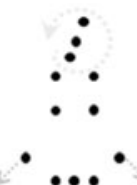

(3)
Fig. 1 Example frames for the biological movement (BM) stimuli (A) and movements of rectangles (B) used in Experiments 1 and 2. A movement of rectangle started from a static dotted-rectangle. When a rectangle began to move, it did the movements illustrated in Fig. 1B (from left to right in turn; the grey arrow was used to illustrate the moving direction of a side and did not appear in the experiment). In particular: (1) The left and right sides moved downward $60^{\circ}$ relative to their vertical positions and then returned. (2) The left side moved upward $45^{\circ}$ relative to its vertical position and then returned, while the right half

presented during each trial. The spatial locations of the stimuli were evenly distributed on the periphery of an invisible circle with a radius of $4.88^{\circ}$ from the screen center.

The color stimuli in Experiment 3 were selected from the following nine colors: red (255, 0,0 , in RGB value), green ( 0 , $255,0)$, blue $(0,0,255)$, yellow $(255,255,0)$, aqua $(0,255$, $255)$, pink $(255,0,255)$, black $(0,0,0)$, white $(255,255,255)$, and dark yellow $(180,180,60)$. Since WM capacity for color is slightly higher than that for BM (e.g., Shen et al., 2014; Vogel, Woodman, \& Luck, 2001), in order to avoid underestimating the WM capacity of colors, each time three to six distinct colors were randomly presented (cf. Rouder, Morey, Morey, \& Cowan, 2011) in Experiment 3. ${ }^{2}$ The spatial locations of the stimuli were randomly located within a radius of $5^{\circ}$ around the screen center.

\section{Procedure}

Participants were told that they would take part in a memory task, and would have to indicate after each trial whether a stimulus appeared in the previous memory set. For

\footnotetext{
$\overline{2}$ Experiment 3 was different from Experiments 1 and 2 in two points in terms of testing procedure: (1) we used different set sizes (3-6 colors for color but $1-5$ for motions); and (2) we did not add an articulatory suppression task in Experiment 3 (see procedure for details). We believe that neither issue contaminated the current findings. In particular, a key issue in the current study was accurately estimating the WM capacity of the tested stimuli. Since the tested stimuli have distinct processing properties in WM (e.g., Shen et al., 2014; Vogel et al., 2001), we had to adjust the tested parameters accordingly. Indeed, the two differences in testing procedure were made based on previous studies which had successfully estimated the WM capacity of corresponding stimuli (e.g., Shen et al., 2014; Vogel et al., 2001).
}

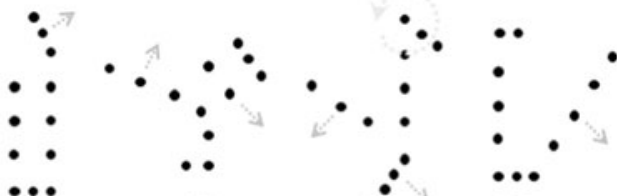

(4)

(5)

(6) $90^{\circ}$. (7) The right side moved downward $45^{\circ}$ and then returned sponse accuracy was emphasized and analyzed.

side moved upward $45^{\circ}$ relative to its vertical position and then returned. (3) The left and right half sides moved downward $90^{\circ}$ relative to their vertical positions and then returned, while the top side rotated around the middle dot once. (4) The top side moved upward $90^{\circ}$ relative to its horizontal position and then returned. (5) The top half rectangle rotated $90^{\circ}$ clockwise relative to its vertical position, while the left bottom half side moved upward $90^{\circ}$. (6) The top side rotated around the top dot in the right side for $180^{\circ}$, while both the left and bottom sides moved downward

Experiments 1 and 2, each trial began by presenting two white digits in the center of the screen (see Fig. 1) for $500 \mathrm{~ms}$ (see Fig. 2A). Participants were required to repeat the two digits (e.g., "nine," "two," "nine," "two") aloud throughout a trial to prevent them from verbally rehearsing the animations (cf. Gao, Bentin, \& Shen, 2014; Shen et al., 2014). Next, a red fixation appeared for $300 \mathrm{~ms}$ to inform the participants of the upcoming memory task. After a blank interval of $150-350 \mathrm{~ms}$, the memory array was presented on the screen for $\mathrm{N} \mathrm{s}$ (according to the number of to-be-memorized stimuli, e.g., $5 \mathrm{~s}$ for 5 stimuli), which was followed by a 1-s blank interval. A red probe was then presented in the screen center for $1 \mathrm{~s}$. At this point participants could stop repeating the digits aloud and were asked to judge whether the stimulus had appeared in the memory array by pressing a button to relay the judgment within a time window of $3 \mathrm{~s}$. Participants were instructed to press " $F$ " on the keyboard if a BM did not appear in the memory set, and "J" if it did appear. After the response, or if no response was made within the time limit, a digit judgment task was presented after a 100-ms delay. Participants had two seconds to determine, by pressing the same buttons, whether the red digit was one of the previously rehearsed digits. Re-

For Experiment 3, we removed the articulatory suppression task since previous studies showed that the verbal rehearsal strategy did not contaminate the WM estimates of color (e.g., Vogel et al., 2001). Each trial began by presenting a dark blue (not used in the memory set) fixation for $300 \mathrm{~ms}$, to inform the participants of the upcoming memory task (see Fig. 2B). After a blank interval of 300-400 ms, the memory array was presented on the screen for $150 \mathrm{~ms}$ (cf. Fukuda \& Vogel, 2009), which was followed by a $900-\mathrm{ms}$ blank interval. A probe was then presented in the center of the screen, and the participants 
A

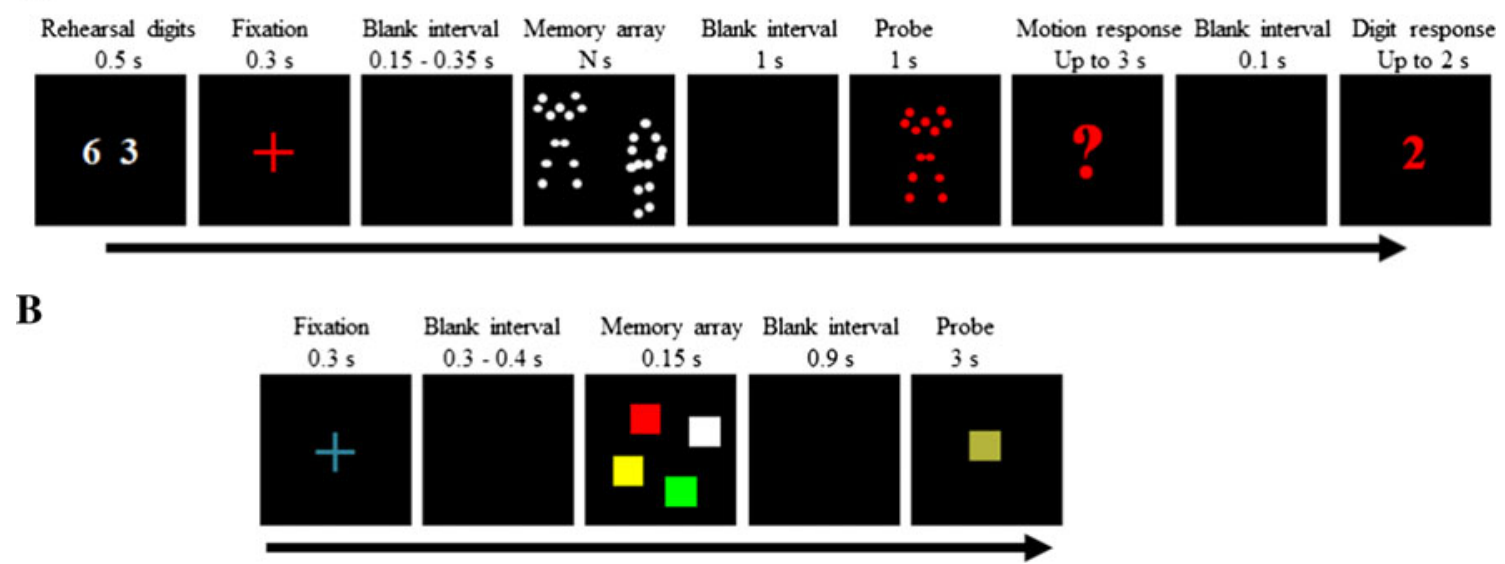

Fig. 2 A schematic representation of a single trial in Experiment 1 (A) and Experiment 3 (B)

judged whether the color had appeared in the memory array, and pressed a button to relay the judgment within a time window of 3 s. Participants were instructed to press " $F$ " on the keyboard if a color did not appear in the memory set and " $\mathrm{J}$ " if it did appear. Response accuracy was emphasized and analyzed.

There were 30 trials under each memory load, resulting in 150 trials for Experiments 1-2 and 120 trials for Experiment 3. Before the formal experiment, the participants practiced at least 16 trials to make sure they were highly familiar with the buttons.

Following the experiments, the participants completed two validated and widely used empathy questionnaires translated into Chinese: the Questionnaire Measure of Emotional Empathy (QMEE; Mehrabian \& Epstein, 1972) and the Empathy Quotient (EQ; Baron-Cohen \& Wheelwright, 2004). The QMEE measures emotional empathy, while the EQ measures empathy in general with a subscale of 11 items that have been shown to tap cognitive empathy skills specifically (Lawrence et al., 2004). Analyzing this subscale in addition to the general questionnaires' scores enabled us to further differentiate between emotional (QMEE) and cognitive empathy, and to examine whether BM-WM capacity was related to empathy in general, or to a specific sub-component.

\section{Analysis}

To estimate WM capacity for each type of stimuli, we employed Cowan's formula (Cowan, 2001): $K=S \times(H-F)$, where $K$ is the WM capacity, $S$ is the number of to-bememorized stimuli, $H$ is the hit rate that refers to the successful detection of a new stimulus, and $F$ is the false alarm rate that refers to an incorrect new-stimulus response. We calculated $K$ for each set size of each participant. To have a more accurate estimate, we considered the maximum $K(K$-max) among the five load conditions as one's WM capacity (e.g., Curby \& Gauthier, 2007; Gao et al., 2014; Shen et al., 2014). For Experiments 1 and 2, only trials with correct digit responses were analyzed. Finally, Pearson's correlations between $K$-max and the scores on the two empathy questionnaires were calculated.

\section{Results}

For the descriptive statistics and the skewness values of $K$-max, QMEE score, EQ score, and cognitive empathy, please see Table 1. One-sample Kolmogorov-Smirnov test showed that all the measured variables conformed to normal distributions ( $p s>.15$; see also the Skewness in Table 1). Additionally, a one-way ANOVA by taking Experiment as a between-subjects variable showed that there was no significant difference among the three experiments on QMEE, $F(2,126)=.402, p=.67$, EQ, $F(2$, $126)=.70, p=.50$, or Cognitive Empathy scores $F(2$, $126)=1.50, p=.23$.

\section{Experiment 1: WM capacity of BM correlates with empathy}

Overall accuracy of the digit rehearsal task was $93 \%$.

Pearson's correlation revealed a significantly positive correlation between $K$-max and the EQ score (Fig. 3A), $r=.337$, $p=.010$, cognitive empathy (Fig. 3B), $r=.311, p=.018$, and the QMEE score (Fig. 3C), $r=.277, p=.037$.

\section{Experiment 2: WM capacity of movements of rectangles does not correlate with empathy}

Overall accuracy of the digit rehearsal task was $97 \%$.

There was no significant correlation between $K$-max and the EQ score (Fig. 3D), $r=.122, p=.478$, cognitive empathy 
Table 1 Mean accuracy (SE) and skewness value of each measured variable used in the current study

\begin{tabular}{llllll}
\hline Experiment & Statistics & WM task $(K$-max $)$ & QMEE Score & EQ Score & Cognitive empathy \\
\hline Experiment 1 & Mean (SE) & $3.00(0.12)$ & $32.95(2.80)$ & $31.86(1.11)$ & $8.75(0.57)$ \\
& Skewness & 0.14 & -0.18 & 0.26 & 0.10 \\
Experiment 2 & Mean (SE) & $2.75(0.13)$ & $37.37(2.96)$ & $31.33(1.39)$ & $7.30(0.72)$ \\
& Skewness & 0.64 & -0.17 & -0.02 & 0.29 \\
Experiment 3 & Mean (SE) & $3.86(0.12)$ & $32.92(3.07)$ & $33.51(1.27)$ & $8.97(0.77)$ \\
& Skewness & -0.26 & -0.12 & -0.13 & 0.15 \\
\hline
\end{tabular}

$S E$ standard error of the mean, $W M$ working memory, $Q M E E$ Questionnaire Measure of Emotional Empathy, $E Q$ emotional quotient

(Fig. 3E), $r=.131, p=.477$, or the QMEE score (Fig. 3F), $r=.049, p=.775$.
(Fig. 3H), $r=.140, p=.408$, or the QMEE score (Fig. 3I), $r=.007, p=.966$.
Experiment 3: WM capacity of colors does not correlate with empathy

There was no significant correlation between $K$-max and the EQ score (Fig. 3G), $r=-.035, p=.837$, cognitive empathy

\section{Discussion}

To the best of our knowledge, the current study is the first study that examines the relationship between empathy and WM directly. The mechanisms of WM are now
Experiment 1

A

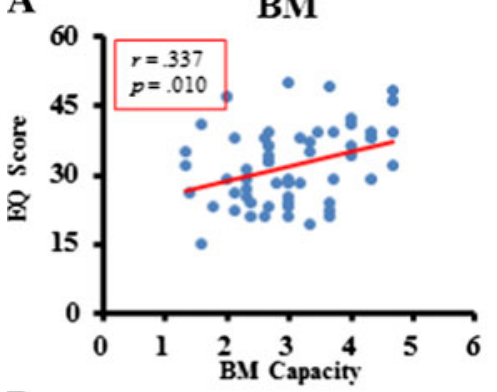

B
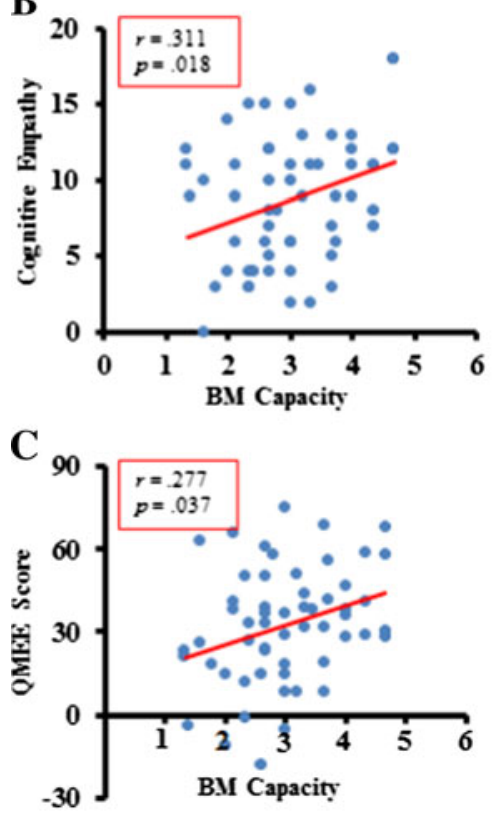

Experiment 2

D

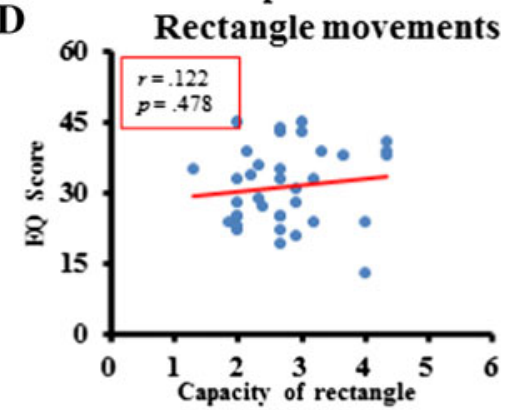

$\mathbf{E}$

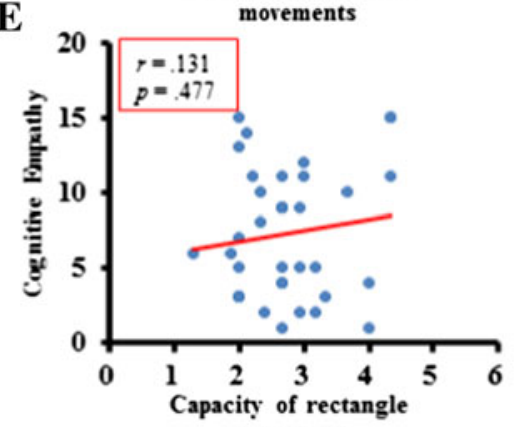

morements

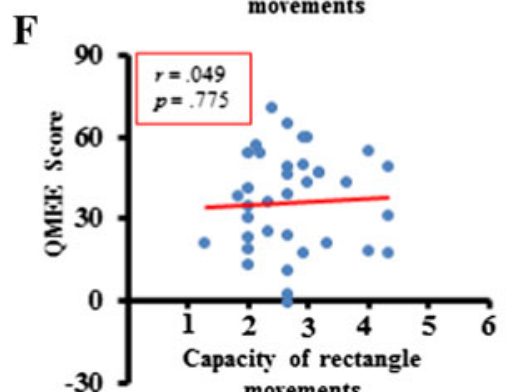

Experiment 3

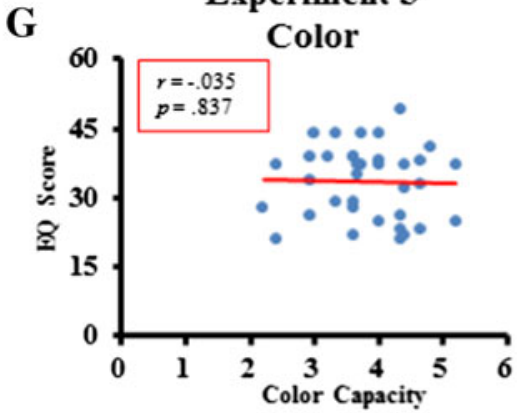

H

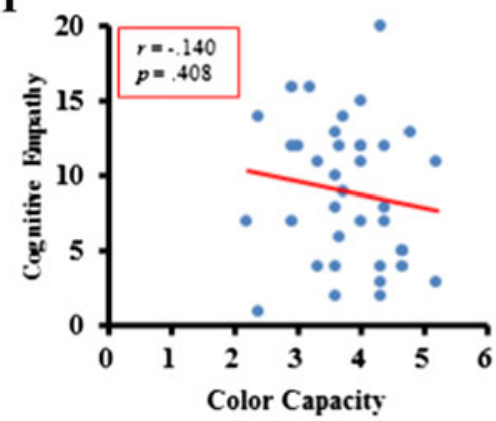

I

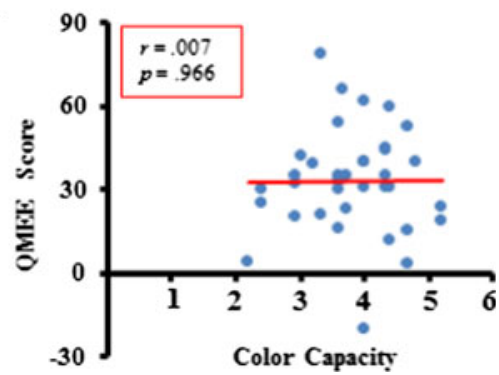

Fig. 3 Results from Experiment 1 (A, B, and C; biological movements (BM)), Experiment 2 (D, E, and F; movements of rectangle), and Experiment 3 (G, $\mathbf{H}$, and $\mathbf{I}$; static colors) 
among the core topics of cognitive science, and the mechanisms underlying empathy are widely studied within the social sciences and neuroscience fields. Although both have received extensive attention, no study has directly linked these two phenomena. The current study is the first to provide evidence of a link between WM capacity for social BM stimuli and one's empathy level.

In line with our prediction, we found a significantly positive correlation between WM capacity and empathy only for BM stimuli. Although the stimuli in Experiments 1 (movements of human beings) and 2 (movements of rectangles) were both dynamic and shared one storage buffer (Shen et al., 2014), a significant correlation was not observed in Experiment 2. Furthermore, although previous studies found that WM capacity for color significantly correlated with fluid intelligence (e.g., Unsworth et al., 2014), we did not observe a correlation between WM for color and empathy (Experiment 3). It is worth noting that we ran Experiment 1 twice with two different groups of participants, and obtained similar findings (see Appendix), suggesting that the results are robust. Therefore, we suggest that WM capacity for BM contributes to our ability to empathize as a buffer that holds social information. Moreover, we found that BMWM capacity was significantly correlated with both cognitive and emotional empathy, suggesting that WM storage is important to both components of empathy. This is reasonable since both in order to feel what the other is feeling and in order to understand the other's intentions and goals, one would benefit from forming a rich coherent representation of the dynamic social scene.

These results add to previous BM perception studies that have linked BM to social abilities (for reviews see Pavlova, 2012; Troje, 2013), and to distinct neural correlates (e.g., Perry et al., 2010a, b; Grezes et al., 2001; Peelen et al., 2006; Saygin et al., 2004; Ulloa \& Pineda, 2007). Moreover, individuals with autistic spectrum disorder (ASD), who have significantly impaired social behavior, have been shown to have deficits in perception of BM (Blake, Turner, Smoski, Pozdol, \& Stone, 2003; Klin, Lin, Gorrindo, Ramsay, \& Jones, 2009); and, most relevant to the current study, Miller and Saygin (2013) demonstrated that one's sensitivity for using form cues in BM processing was significantly correlated with the EQ in a healthy population. The current study for the first time implies that WM capacity of BM has a relationship with the social abilities of human beings.

It should be noted that since we measured correlations, we cannot rule out an alternative interpretation,

A

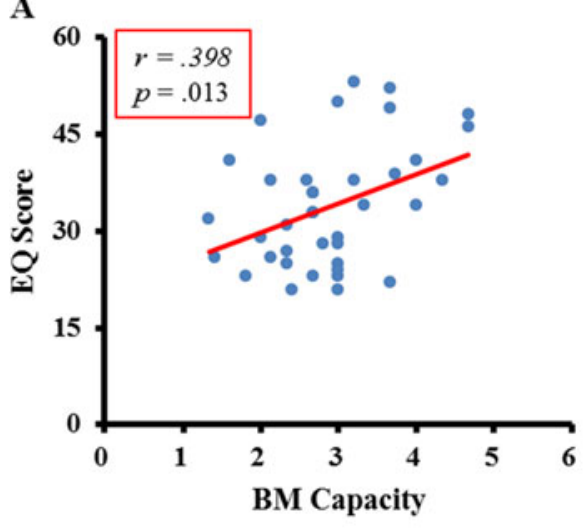

B

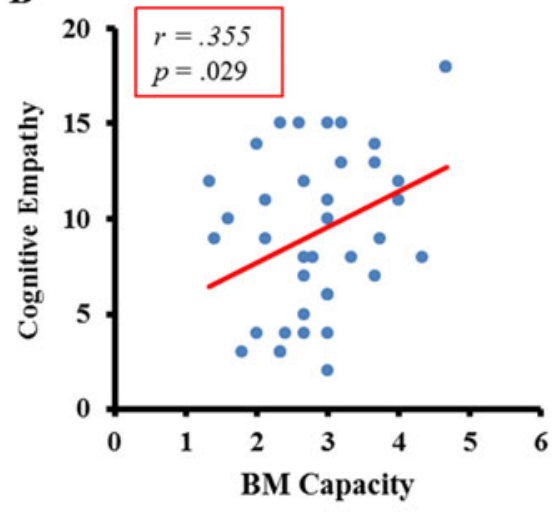

C

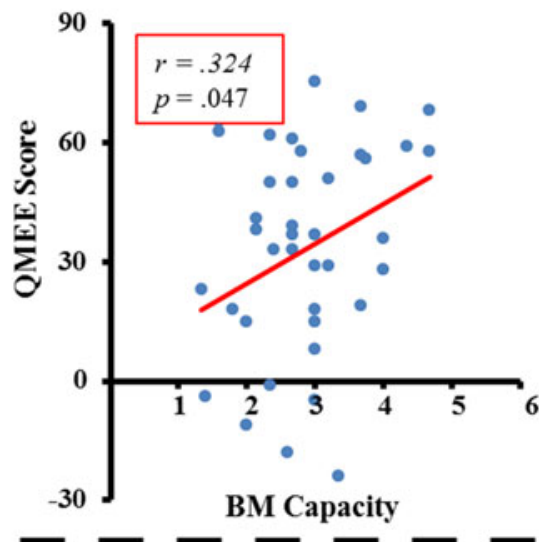

D

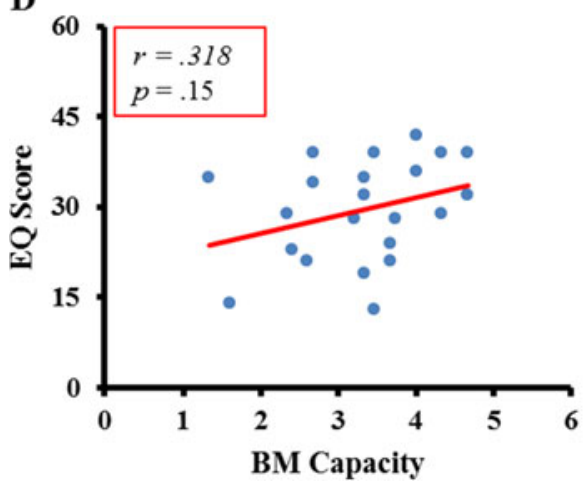

E

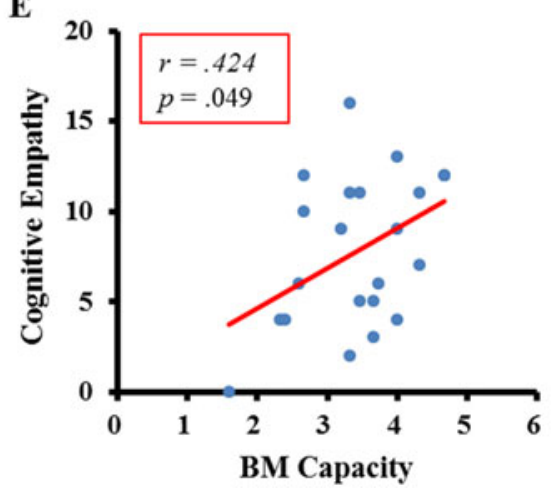

F

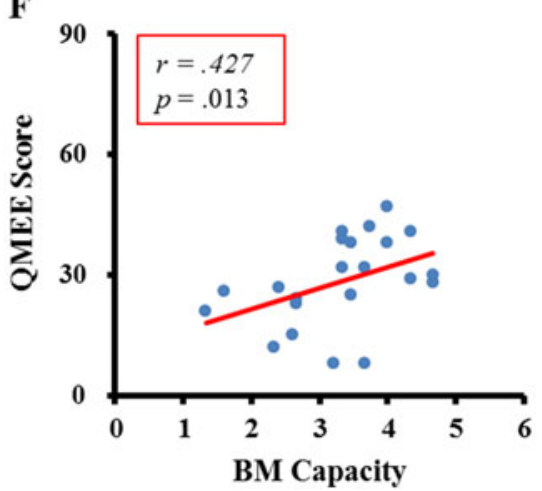

Fig. 4 Results for the first 40 participants (A, B \& C), and for the last 26 participants (D, E \& F) 
which suggests that one's empathy level elevates social WM capacities, or in fact, that both mechanisms are correlated because they stem from a common trait (e.g., a broader trait of "emotional intelligence," Salovey \& Mayer, 1989). Future studies are needed to determine the causal role between these two constructs.

The current results may have important implications for the future clinical diagnosis of social-functioning deficits. Researchers and clinicians have long realized the importance of examining WM functioning in patients (e.g., Parkinson disease, schizophrenia), and showed that both WM capacity and the ability to filter irrelevant information from WM were impaired in these groups (e.g., Lee et al., 2010; Leonard et al., 2012). However, these studies adopted common objects (e.g., color) and not BM as stimuli. While there is abundant literature on BM perception deficits in ASD (e.g., Blake et al., 2003; Klin et al., 2009; Van Boxtel \& Lu, 2013), no study has examined WM mechanisms involved in BM information in clinical populations. For example, it may be the case that WM for BM is specifically impaired in patients with known social deficits (e.g., autism, schizophrenia), but is elevated in patients with social anxiety disorder who in some cases have been shown to pay more attention to social stimuli (e.g., Mogg \& Bradley, 2002). Furthermore, some patients may show a dissociation between their WM capacities for social versus non-social stimuli. For instance, people with ASD may show a greater than average capacity for memorizing colors or shapes, with a lower WM capacity for BM (e.g., Richmond, Thorpe, Berryhill, Klugman, \& Olson, 2013). Lastly, the link between WM capacities and social functioning may open a venue for social interventions focused on WM, which will in turn enhance social understanding.

Acknowledgments We thanked two anonymous reviewers for their constructive comments on an earlier draft. This research is supported by the Natural Science Foundation of China (NSFC; No. 31271089 and 31170975).

\section{Appendix}

\section{WM capacity of BM correlates with empathy (first 40 participants)}

Forty participants ( 16 females) took part in the experiment, 2 participants (1 male) were removed because QMEE score was beyond 2 standard deviations.

Pearson's correlation revealed a significantly positive correlation between $K$-max and the EQ score (Fig. 4), $r=.398, p=.013$, cognitive empathy (Fig. 4B), $r=.355$, $p=.029$, and the QMEE score (Fig. 4C), $r=.324$, $p=.047$.

\section{WM capacity of BM correlates with empathy (26 participants after 6 months)}

Twenty-six new participants ( 9 females) participated in this part of experiment.

Three participants ( 2 males) were removed because the empathy score was beyond 2 standard deviations, 1 male participant was removed because of too low behavioral performance of BM.

Pearson's correlation also revealed a significantly positive correlation between $K$-max and the QMEE score (Fig. 4F), $r=.427, p=.013$, and cognitive empathy (Fig. 4E), $r=.424$, $p=.049$. The correlation between K-max and EQ was not significant, $r=.318, p=.15$, yet a similar trend as QMEE was revealed (Fig. 4D).

The non-significant correlation between $K$-max and EQ may be due to the variance in the participants since only 22 valid participants were analyzed.

\section{References}

Baddeley, A. (2003). Working memory and language: An overview. Journal of Communication Disorders, 36(3), 189-208.

Baddeley, A. (2012). Working memory: Theories, models, and controversies. Annual Review of Psychology, 63(1), 1-29.

Baird, A. D., Scheffer, I. E., \& Wilson, S. J. (2011). Mirror neuron system involvement in empathy: a critical look at the evidence. Socical Neuroscience, 6(4), 327-335.

Baron-Cohen, S., \& Sally, W. (2004). The empathy quotient: an investigation of adults with Asperger syndrome or high functioning autism, and normal sex differences. Journal of Autism and Developmental Disorders, 34(2), 163-175.

Batson, C. D., Polycarpou, M. P., Harmon-Jones, E., Imhoff, H. J., Mitchener, E. C., Bednar, L. L., ... \& Highberger, L. (1997). Empathy and attitudes: Can feeling for a member of a stigmatized group improve feelings toward the group?. Journal of personality and social psychology, 72(1), 105.

Blake, R., Turner, L. M., Smoski, M. J., Pozdol, S. L., \& Stone, W. L. (2003). Visual recognition of biological motion is impaired in children with autism. Psychological Science, 14, 151-157.

Blakemore, S. J. (2008). The social brain in adolescence. Nature Reviews. Neuroscience, 9(4), 267-277.

Cowan, N. (2001). The magical number 4 in short-term memory: a reconsideration of mental storage capacity. Behavioral and Brain Sciences, 24(1), 87-114.

Cowan, N., Elliott, E. M., Scott Saults, J., Morey, C. C., Mattox, S., Hismjatullina, A., \& Conway, A. R. (2005). On the capacity of attention: its estimation and its role in working memory and cognitive aptitudes. Cognitive Psychology, 51(1), 42-100.

Curby, K. M., \& Gauthier, I. (2007). A visual short-term memory advantage for faces. Psychonomic Bulletin \& Review, 14(4), 620-628.

Decety, J., \& Jackson, P. L. (2004). The functional architecture of human empathy. Behavioral and Cognitive Neuroscience Reviews, 3(2), $71-100$.

Eslinger, P. J. (1998). Neurological and neuropsychological bases of empathy. European Neurology, 39, 193-199.

Fukuda, K., \& Vogel, E. K. (2009). Human variation in overriding attentional capture. Journal of Neuroscience, 29(27), 8726-8733. 
Gao, Z., Bentin, S., \& Shen, M. (2014). Rehearsing biological motion in working memory: An EEG study. Journal of Cognitive Neuroscience, 27(1), 198-209.

Grezes, J., Fonlupt, P., Bertenthal, B., Delon-Martin, C., Segebarth, C., \& Decety, J. (2001). Does perception of biological motion rely on specific brain regions? NeuroImage, 13(5), 775-785.

Henderson, J. M., \& Hollingworth, A. (2003). Eye movements, visual memory, and scene representation. Perception of faces, objects and scenes: Analytic and holistic processes, 356-383

Johansson, G. (1973). Visual-perception of biological motion and a model for its analysis. Perception \& Psychophysics, 14(2), 201-211.

Klin, A., Lin, D. J., Gorrindo, P., Ramsay, G., \& Jones, W. (2009). Twoyear-olds with autism orient to non-social contingencies rather than biological motion. Nature, 459, 257-261.

Knafo, A., Zahn-Waxler, C., Van Hulle, C., Robinson, J. L., \& Rhee, S. H. (2008). The developmental origins of a disposition toward empathy: Genetic and environmental contributions. Emotion, 8(6), 737.

Lawrence, E. J., Shaw, P., Baker, D., Baron-Cohen, S., \& David, A. S. (2004). Measuring empathy: reliability and validity of the Empathy Quotient. Psychological Medicine, 34, 911-924.

Lee, E. Y., Cowan, N., Vogel, E. K., Rolan, T., Valle-Inclan, F., \& Hackley, S. A. (2010). Visual working memory deficits in patients with Parkinson's disease are due to both reduced storage capacity and impaired ability to filter out irrelevant information. Brain, 133(9), 2677-2689.

Leonard, C. J., Kaiser, S. T., Robinson, B. M., Kappenman, E. S., Hahn, B., Gold, J. M., \& Luck, S. J. (2012). Toward the neural mechanisms of reduced working memory capacity in schizophrenia. Cerebral Cortex

Luck, S. J., \& Vogel, E. K. (2013). Visual working memory capacity: from psychophysics and neurobiology to individual differences. Trends in Cognitive Sciences, 17(8), 391-400.

Mance, I., \& Vogel, E. K. (2013). Visual working memory. Wiley Interdisciplinary Reviews: Cognitive Science, 4(2), 179-190.

Mehrabian, A., \& Epstein, N. (1972). A measure of emotional empathy. Journal of Personality, 40(4), 525-543.

Miller, L. E., \& Saygin, A. P. (2013). Individual differences in the perception of biological motion: Links to social cognition and motor imagery. Cognition, 128(2), 140-148.

Mogg, K., \& Bradley, B. P. (2002). Selective orienting of attention to masked threat faces in social anxiety. Behaviour Research and Therapy, 40(12), 1403-1414.

Pavlova, M. (2012). Biological motion processing as a hallmark of social cognition. Cerebral Cortex, 22(5), 981-995. doi:10.1093/cercor/ bhr156

Peelen, M. V., Wiggett, A. J., \& Downing, P. E. (2006). Patterns of fMRI activity dissociate overlapping functional brain areas that respond to biological motion. Neuron, 49(6), 815-822.

Perry, A., Bentin, S., Shalev, I., Israel, S., Uzefovsky, F., Bar-On, D., \& Ebstein, R. P. (2010a). Intranasal oxytocin modulates EEG mu/alpha and beta rhythms during perception of biological motion. Psychoneuroendocrinology, 35(10), 1446-1453.

Perry, A., Troje, N. F., \& Bentin, S. (2010b). Exploring motor system contributions to the perception of social information: Evidence from EEG activity in the mu/alpha frequency range. Social Neuroscience, 5(3), 272-284

Richmond, L. L., Thorpe, M., Berryhill, M. E., Klugman, J., \& Olson, I. R. (2013). Individual differences in autistic trait load in the general population predict visual working memory performance. Quarterly Journal of Experimental Psychology, 66(6), 1182-1195.

Rouder, J. N., Morey, R. D., Morey, C. C., \& Cowan, N. (2011). How to measure working memory capacity in the change detection paradigm. Psychonomic Bulletin \& Review, 18(2), 324-330.

Salovey, P., \& Mayer, J. D. (1989). Emotional intelligence. Imagination, Cognition and Personality, 9(3), 185-211.

Saygin, A. P., Wilson, S. M., Hagler, D. J., Bates, E., \& Sereno, M. I. (2004). Point-light biological motion perception activates human premotor cortex. The Journal of Neuroscience, 24(27), 6181-6188.

Shamay-Tsoory, S. G. (2011). The neural bases for empathy. The Neuroscientist, 17(1), 18-24.

Shamay-Tsoory, S. G., Tomer, R., Goldsher, D., Berger, B. D., \& AharonPeretz, J. (2004). Impairment in cognitive and affective empathy in patients with brain lesions: Anatomical and cognitive correlates. Journal of Clinical and Experimental Neuropsychology, 26(8), $1113-1127$.

Shen, M., Gao, Z., Ding, X., Zhou, B., \& Huang, X. (2014). Holding Biological Motion Information in Working Memory. Journal of Experimental Psychology. Human Perception and Performance, 40(4), 1332-1345.

Simmons, J. P., Nelson, L. D., \& Simonsohn, U. (2011). False-positive psychology undisclosed flexibility in data collection and analysis allows presenting anything as significant. Psychological Science, 22(11), 1359-1366.

Smyth, M. M., \& Pendleton, L. R. (1990). Space and movement in working memory. The Quarterly Journal of Experimental Psychology, 42(2), 291-304.

Troje, N. F. (2013). What is biological motion?: Definition, stimuli and paradigms. In M. D. Rutherford \& V. A. Kuhlmeier (Eds.), Social perception: Detection and interpretation of animacy, agency, and intention (pp. 13-37): MIT Press.

Ulloa, E. R., \& Pineda, J. A. (2007). Recognition of point-light biological motion: mu rhythms and mirror neuron activity. Behavioural Brain Research, 183(2), 188-194.

Unsworth, N., Fukuda, K., Awh, E., \& Vogel, E. K. (2014). Working memory and fluid intelligence: Capacity, attention control, and secondary memory retrieval. Cognitive Psychology, 71, 1-26.

Urgolites, Z. J., \& Wood, J. N. (2013). Visual long-term memory stores high-fidelity representations of observed actions. Psychological Science, 24(4), 403-411.

van Boxtel, J. J. A., \& Lu, H. (2013). Impaired global, and compensatory local, biological motion processing in people with high levels of autistic traits. Frontiers in Psychology. doi:10.3389/fpsyg.2013. 00209

Vanrie, J., \& Verfaillie, K. (2004). Perception of biological motion: a stimulus set of human point-light actions. Behavior Research Methods, Instruments, \& Computers, 36(4), 625-629.

Vogel, E. K., Woodman, G. F., \& Luck, S. J. (2001). Storage of features, conjunctions and objects in visual working memory. Journal of Experimental Psychology. Human Perception and Performance, 27(1), 92-114.

Wood, J. N. (2007). Visual working memory for observed actions. Journal of Experimental Psychology. General, 136(4), 639-652.

Zaki, J. (2014). Empathy: A motivated account. Psychological Bulletin, 140(6), 1608-1647. 